\title{
Bilateral pulmonary embolism and ilio-femoral DVT associated with recent amputation of lower limb, long distance air travel and suspected thrombophilia.
}

Mohammad Omar Faruq1, Syed Muhammad Shahin-ur Hayat², Rezaul Karim², Tahsin Salam², Aslam Uddin Sardar², Faisal Kabir ${ }^{2}$, Arifur Rahman Khan², Dewan Muhammad Mahmudul Gafur², Mohammad Syam², A K M Nahid Arefin², Shamima Choudhury ${ }^{3}$

\begin{abstract}
:
We present a 36 years old Bangladeshi male, known smoker, while working in Bahrain, suffered from arterial thromboembolism on left lower extremity resulting in gangrene of left leg. He underwent above knee amputation of the affected limb. After 16 days stay in the hospital with an open amputated stump wound, he was sent back to Bangladesh by air. While in the airplane, he complained of chest discomfort about two hours before landing at Dhaka airport of Bangladesh. Following disembarkation he was admitted into local cardiac hospital where a diagnosis of left ventricular failure with unstable angina was made. Five days later he was transferred to ICU of Ibn Sina Hospital for better management. Patient had high serum D-dimer level and fibrin degradation products (FDP) level. negative antinuclear antibody (ANA) test, negative anti-cardiolipin antibody test, normal troponin I, Homocysteine, antithrombin III, protein $S, \&$ protein $C$ levels. Initial X-ray chest showed left lower zone wedge shaped density. ECG showed sinus tachycardia. CT angiogram of chest showed bilateral pulmonary embolism (PE) and large left pleural effusion. Contrast CT abdomen showed bilateral iliac vein thrombus extending to lower inferior vena cava. Left pleural effusion was found to be grossly hemorrhagic. Patient was treated with low molecular weight heparin and warfarin. As thrombolysis was not feasible, he was advised to have thrombo-embolectomy. He refused surgical option and left hospital against medical advice.
\end{abstract}

This case illustrates that multiple risk factors can be responsible for PE, and appropriate \& timely interventions are always needed to prevent morbidity and or mortality.

Key words : Pulmonary embolism, Deep vein thrombosis, Risk factors, Venous thrombo embolism.

\section{Introduction :}

Pulmonary embolism (PE) and deep vein thrombosis (DVT) represent the spectrum of one disease ${ }^{1}$. It is one of the commonest non-diagnosed or misdiagnosed diseases found in literature. Many of the symptoms and signs detected in patients in $\mathrm{PE}$ are common among patients without $\mathrm{PE}$ making the diagnosis of PE clinically difficult. Clinical severity of $\mathrm{PE}$ can be highly variable ranging from asymptomatic to severe hypoxemia and shock ${ }^{2}$.

$\mathrm{PE}$ originates in the deep veins of legs, most commonly calf veins. These venous thrombi originate predominantly in the venous valve-pockets and in other sites of presumed venous stasis. If a clot propagates to the knee vein or above or if it originates above the knee, the risk of embolism increases ${ }^{1}$. About $79 \%$ of patients who present with PE have evidence of DVT in their legs ${ }^{3}$.

1. Prof of Critical Care Medicine, Ibn Sina hospital, Dhaka Bangladesh.

2. Medical Officer, Ibn Sina Hospital, Dhaka, Bangladesh.

3. Lecturer, Green Life Hospital, Dhaka, Bangladesh.

Corresponding Author :

Prof Mohammad Omar Faruq MD, FACP, FACEP, FCPS, FCCM

Professor of Critical Care Medicine

Ibn Sina Hospital

E mail: faruqmo@yahoo.com
$\mathrm{PE}$ is an acute and potentially fatal condition in which embolic material, usually a thrombus from veins of legs or pelvis blocks one or more pulmonary arteries causing impaired blood flow and increased pressure to the right ventricle ${ }^{4}$.

In 1856, Rudolph Virchow first proposed that thrombosis was the result of at least one of three underlying etiologic factors: vascular endothelial damage, stasis of blood flow and hyper coagulability of blood ${ }^{5}$.

Risk factors of venous thrombo embolism (VTE) include hereditary factors like deficiency of protein $\mathrm{C}$ and $\mathrm{S}$ and deficiency of anti thrombin. However acquired risk factors are more common such as reduced mobility, advancing age, cancer, major surgery, trauma, antiphospholipid antibody syndrome, oral contraceptive, pregnancy, hormone replacement therapy, obesity, central venous cannulation etc ${ }^{1}$.

In a review of 1231 consecutive patients treated for VTE, Andersen et $\mathrm{al}^{6}$ found distribution of risk factors as follows : $88.5 \%$ were $\geq 40$ years of age, $37.8 \%$ were obese, $26 \%$ had history of VTE, $22.3 \%$ had cancer, $12 \%$ had bed rest $\geq 5$ days, $11.2 \%$ had major surgery, $8.2 \%$ had congestive heart failure, $5.8 \%$ had varicose veins, $3.7 \%$ had hip or leg fracture, $2 \%$ had estrogen treatment, $1.8 \%$ had stroke, $1.1 \%$ had multiple trauma, $1.1 \%$ had child birth and $0.7 \%$ had myocardial infarction. The authors also observed that overall $96 \%$ of study VTE patients had one or more risk factors. 
According to one literature risk factors for PE are classified according to odds ratio justifying heparin prophylaxis against VTE into strong, moderate and weak risk factors ${ }^{7}$. Strong risk factors with odds ratio $>10$ include fracture of hip or leg, hip or knee replacement, major general surgery, major trauma and spinal cord surgery. Moderate risk factors with odds ratio 2-9 include arthroscopic knee surgery, central venous line, malignancy, oral contraceptive therapy, pregnancy post partum, previous VTE, thrombophilia etc. Weak risk factors with odds ratio less than 2 include bed rest $>3$ days, prolonged car/air travel, laparoscopic surgery, pregnancy anti partum, varicose veins etc.

\section{Case note :}

36 year old Bangladeshi male smoker (11-13 pack a year for 22 years) with negative history of diabetes mellitus and intravenous drug abuse presented to ICU of Ibn Sina hospital on Jan 302015 with diagnosis of left ventricular failure, unstable angina, pneumonia and status post above knee amputation of left lower extremity. About 3 weeks prior to admission while working in Bahrain as an expatriate Bangladeshi worker he complained of sudden onset of weakness and tingling sensation in both legs. The symptoms were followed by bluish discoloration of skin of left leg and toes with severe pain of left leg in the next few hours. He was diagnosed to have arterial thromboembolism of left lower extremity causing ischemia of left lower limb. An emergency embolectomy with end-arterectomy were done with patch grafts on two consecutive settings, but the left leg ischaemia showed no improvement. Two days later above knee amputation of left lower extremity was done with wound of amputation stump being kept open. He needed daily dressing and was treated with low molecular weight heparin.

On Jan 25, 2015, after being discharged from Bahrain hospital he was transported to board airplane to travel to Bangladesh. It was about $4000 \mathrm{~km}$ distance by air toward his destination. And including transit time, it took him nine hours to reach Dhaka airport. About two hours prior to reaching Dhaka he complained of severe pain on the amputated stump and chest discomfort. So on disembarkation from plane he was admitted into coronary care unit of a cardiac hospital of Dhaka. A diagnosis of unstable angina and left ventricular failure was made on admission there. The exposed wound of amputated above knee stamp was found to have purulent discharge (Fig 1). He was febrile and complained of palpitation, cough and chest pain. Five days later he was transferred to ICU of Ibn Sina Hospital.

In 2008 (i.e. 7 years prior to this admission) patient was treated in a Bangladeshi hospital for unexplained DVT of left leg, right sided pneumonia with pleural effusion. No information was available on the nature of the pleural fluid at that time. $\mathrm{He}$ was placed on warfarin tablet. A year later he went to India for advanced treatment of DVT and was advised to have placement of inferior vena cava filter but he declined. There was no family history of peripheral vascular disease or VTE.

On admission into ICU of Ibn Sina Hospital he was found to have normal blood pressure with peripheral pulse 130/min, temp $100^{\circ} \mathrm{F}$, respiration $20 / \mathrm{min}$. Arterial blood gas in room air was suggestive of acute type-II respiratory failure and slight hypoxaemia. But hypoxaemia was corrected with supplemental nasal oxygen and he received intermittent non invasive ventilation. The following worth mentioning lab tests were performed during the first few days of admission (Table 1).

Table -1

\begin{tabular}{|c|c|c|}
\hline Tests & Result & Reference Value \& Unit \\
\hline $\mathrm{Hb}$ & $10.2 \mathrm{gm} / \mathrm{dl}$ & $\mathrm{M}: 15+2, \mathrm{~F}: 13.5+1.5 \mathrm{gm} / \mathrm{dl}$ \\
\hline WBC & $17780 / \mathrm{cmm}, 76 \%$ poly & 4,000-11,000/cumm \\
\hline ESR & $82 \mathrm{~mm}$ in 1 st hour & $\mathrm{M}: 0-10, \mathrm{~F}: 0-20 \mathrm{~mm}$ in $1 \mathrm{st}$ hou \\
\hline Platelet & $262,000 / \mathrm{cmm}$ & $1,50,000-4,00,000 / \mathrm{cmm}$ \\
\hline RBS & $5.00 \mathrm{mmol} / \mathrm{L}$ & $\mathrm{mmol} / \mathrm{L}$ \\
\hline Urea & $29 \mathrm{mg} / \mathrm{dl}$ & $15-40 \mathrm{mg} / \mathrm{dl}$ \\
\hline Creatinine & $1 \mathrm{mg} / \mathrm{dl}$ & $0.6-1.3 \mathrm{mg} / \mathrm{dl}$ \\
\hline Billirubin & $0.77 \mathrm{mg} / \mathrm{dl}$ & $0.0-1.0 \mathrm{mg} / \mathrm{dl}$ \\
\hline SGOT & $72 \mathrm{U} / \mathrm{L}$ & $\mathrm{M}-<37, \mathrm{~F}-<31 \mathrm{U} / \mathrm{L}$ \\
\hline SGPT & $120 \mathrm{U} / \mathrm{L}$ & $\mathrm{M}-<42, \mathrm{~F}-<31 \mathrm{U} / \mathrm{L}$ \\
\hline Alk Phos & 170 & 98-279 U/L \\
\hline T.Protein & $7.8 \mathrm{gm} / \mathrm{dl}$ & $6.4-8.3 \mathrm{gm} / \mathrm{dl}$ \\
\hline S. Albumin & $2.6 \mathrm{gm} / \mathrm{dl}$ & $3.8-4.4 \mathrm{gm} / \mathrm{dl}$ \\
\hline Trop-1 & $<0.03 \mathrm{ng} / \mathrm{ml}$ & AMI: $0.4 \mathrm{ng} / \mathrm{ml}$ \\
\hline PT-C/patient & $12 \mathrm{sec} / 12 \mathrm{sec}$ & \\
\hline APTT-C/patient & $30 \mathrm{sec} / 24 \mathrm{sec}$ & \\
\hline D-Dimer & $3200 \mathrm{ng} / \mathrm{ml}$ & $<200 \mathrm{ng} / \mathrm{ml}$ \\
\hline FDP & $40 \mathrm{mg} / \mathrm{ml}$ & $<5 \mathrm{mg} / \mathrm{ml}$ \\
\hline Antithrombin -III & $91 \%$ & $75-125 \%$ \\
\hline Anti Cardiolipin antibody & Reported as Negative & \\
\hline Uric acid & $4.4 \mathrm{mg} / \mathrm{dl}$ & $3.5-7.2 \mathrm{mg} / \mathrm{dl}$ in male \\
\hline S. Electrolyte & Normal range & \\
\hline S Magnesium & $2.1 \mathrm{mg} / \mathrm{dl}$ & $0.7-1.1 \mathrm{mg} / \mathrm{dl}$ \\
\hline Calcium & $8.3 \mathrm{mg} / \mathrm{dl}$ & $8.2-10.2 \mathrm{mg} / \mathrm{dl}$ \\
\hline P04 & $3.4 \mathrm{mg} / \mathrm{dl}$ & $2.5-4.5 \mathrm{mg} / \mathrm{dl}$ \\
\hline S Protein C & $88 \%$ & $70-120 \%$ \\
\hline S Protein S & $93 \%$ & $60-100 \%$ \\
\hline ANA & Negative & \\
\hline CRP & $96 \mathrm{mg} / \mathrm{dl}$ & Upto $8.6 \mathrm{mg} / \mathrm{dl}$ \\
\hline
\end{tabular}

Culture studies done on blood, urine, sputum and discharge from amputated stump yielded no growth. Admission ECG showed mild tachycardia and non specific ST-T changes. However ECG on discharge showed T inversion in Lead II, III \& AVF. 


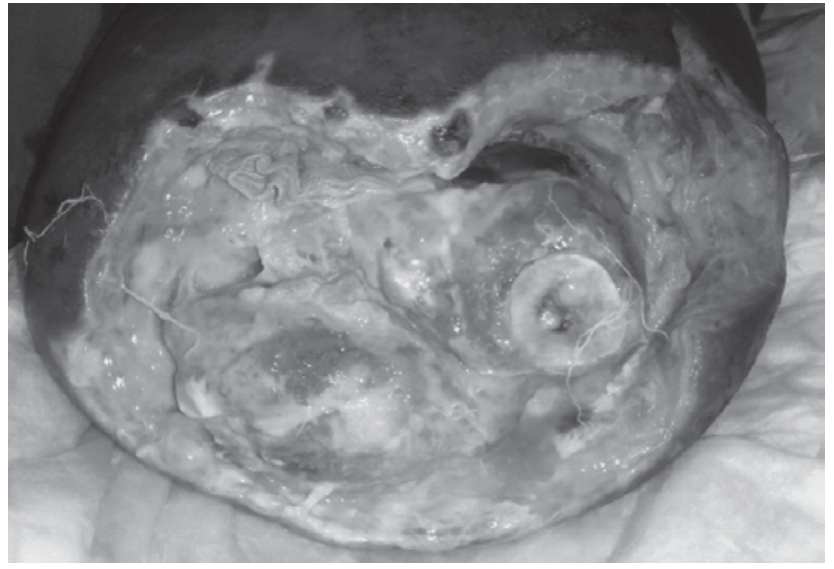

Fig-1

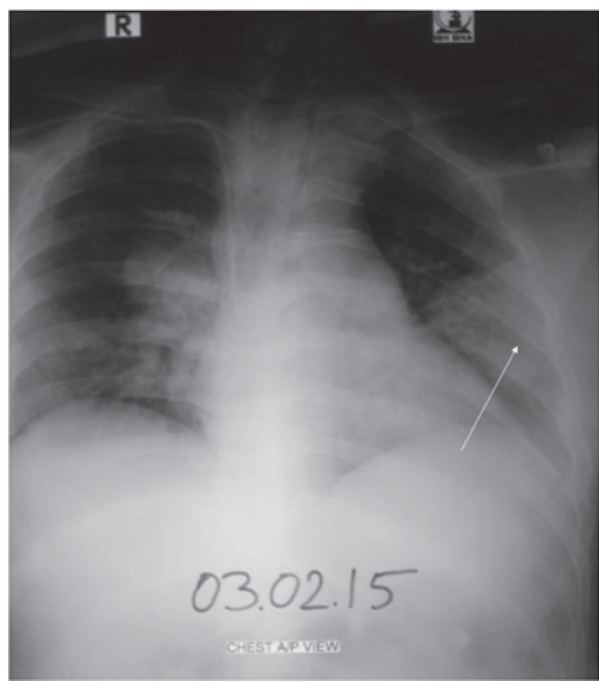

Fig-2

Admission portable chest X-ray showed small infiltrate on left lower lung field but three days later it grew into a wedge shaped well defined density in left lower lung field (Fig-2 arrow). Bed side $M$ mode Echocardiogram showed ejection fraction (EF) of $63 \%$ and no regional wall motion abnormalities. Presence of pulmonary hypertension could not be established as color Doppler Echo study was not available at that time.

A duplex scan of right lower limb showed normal veins but no blood flow in right anterior tibial artery and arterio dorsalis pedis artery. Ultrasonography of abdomen was suggestive of cholelithiasis.

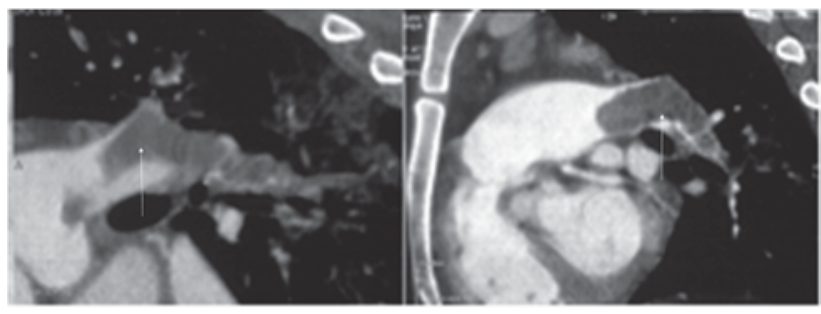

Fig-3

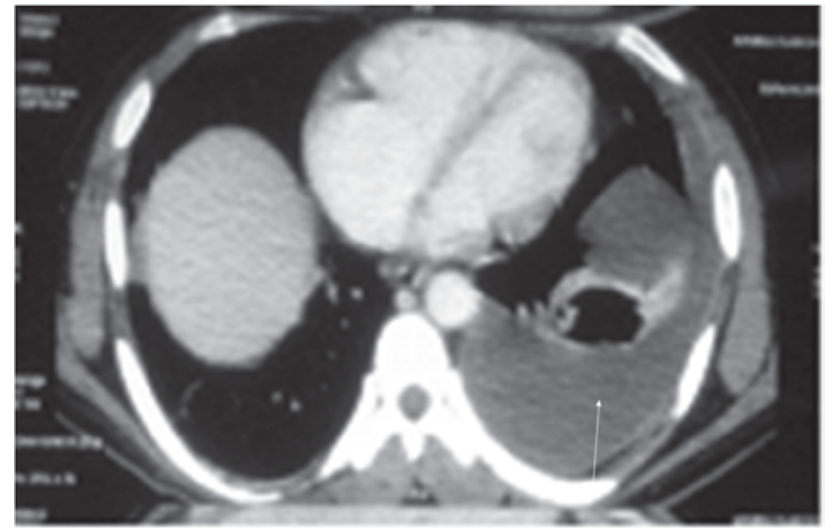

Fig- 4

Five days after admission CT angiogram of pulmonary arteries revealed thrombus on right and left pulmonary arteries (Fig-3 arrows) and a large pleural effusion in left side (Fig-4 arrow)

During the course of stay in ICU patient had persistent tachycardia (130-140/min) and tachypnea (30-40/min) and he was always on supplemental oxygen by nasal cannula.

Nine days after admission, contrast CT scan of abdomen revealed thrombus in right and left iliac veins extending to lower end of inferior vena cava (IVC) (Fig-5 arrow).

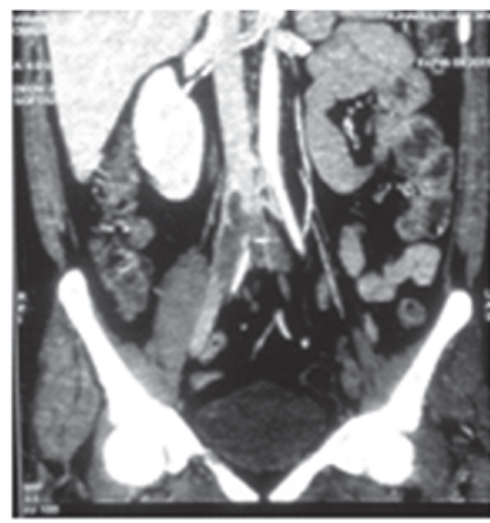

Fig-5

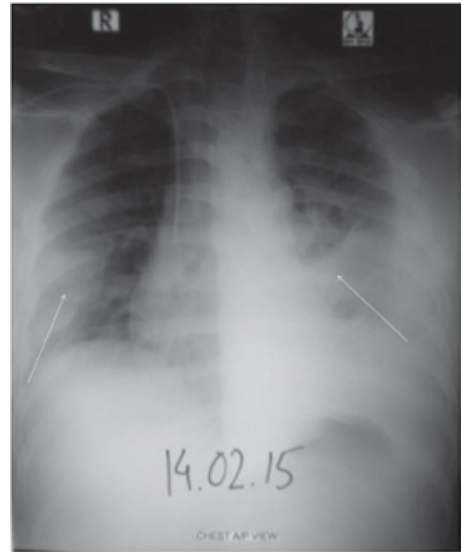

Fig-6 
Fifteen days after admission $\mathrm{x}$-ray chest revealed a new wedge shaped infiltrate on right lower chest in addition to the wedge shaped density noted on the left chest before (Fig-6 arrows). Left pleural effusion was aspirated and it was found to be frankly hemorrhagic.

In addition to administration of low molecular weight heparin (enoxaparin) patient was treated with broad spectrum antibiotics, omeprazole, clopidogrel, tramadol. Wound of amputated stump was treated with application of topical honey, followed by regular dressing. Warfarin tablet was added to enoxaparin five days after admission. Thrombolysis was not considered for our patient because of risk of bleeding. He was seen by cardiovascular surgeon who advised thrombo embolectomy. Patient refused surgical option and opted to be transferred out of ICU. He eventually went home and lost contact with us.

\section{Discussion :}

This 31 year old Bangladeshi male presented with DVT of left lower limb seven years prior to this admission. He was a heavy smoker for last 22 years. His current morbidity began with acute left femoral artery occlusion resulting in gangrene of left leg requiring above knee amputation. Previous history of DVT, major surgery of left lower limb followed by prolonged lying down in hospital in Bahrain and subsequent long distance air travel appear as significant risk factors for PE in this patient ${ }^{1}$. History of recurrent DVT in leg and arterial thromboembolism of lower limb raises strong suspicion of acquired thrombophilia like primary anti phospholipid syndrome ${ }^{8-9}$. However his anticardiolipin antibody test was found negative. Lupus anticoagulant test and test for Anti ß-2 glycoprotein I were not available. Patient had no clinical findings of Systemic lupus erythematosus (SLE) and ANA test was found negative.

He had high serum level of D-dimer which strongly suggested the probability of PE. According to Righini et $\mathrm{al}^{10}$, in patient with high probability of PE imaging should be performed even if the D-dimer test is found negative. In our patient CT angiogram of pulmonary arteries confirmed presence of PE.

Although a positive D-dimer test (which measures plasma level of specific derivative of cross-linked fibrins) indicates that venous thrombus and $\mathrm{PE}$ are possible diagnosis, this test is nonspecific since it may be positive in patients with infection, cancer, trauma and other inflammatory states ${ }^{11}$. D-dimer testing is best considered together with clinical probability and the later can be estimated on the basis of one of two clinical prediction scores: Wells prediction score ${ }^{12}$ and revised Geneva Score ${ }^{13}$. Our patient's Wells score clinical probability was high (with score of 10.5) and revised Geneva Score clinical probability was intermediate (with score of 10)

Until recently PE has been classified into two groups: massive or non-massive based on presence or absence of cardiogenic shock. Contemporary management however involves the identification of an intermediate high risk group of patients with right ventricular dysfunction and / or necrosis with preserved systolic arterial pressure and it is termed as sub massive $\mathrm{PE}^{14}$. Our patient did not have documented shock or hypotension during the course of morbidity. So PE in this case was presumed but not confirmed to be of sub massive type as we could not establish right ventricular dysfunction and pulmonary hypertension because of lack of color Doppler Echo. Myocardial necrosis was however ruled out by a normal troponin I level.

The ECG showed nonspecific ST- T changes with sinus tachycardia on admission and subsequent ECG showed $\mathrm{T}$ wave inversion in inferior leads. ECG changes of acute cor pulmonale such as $\mathrm{S} 1, \mathrm{Q} 3, \mathrm{~T} 3$ pattern, $\mathrm{RBBB}, \mathrm{P}$ pulmonale or right axis deviation are more common with massive $\mathrm{PE}$ than smaller emboli. But these findings are nonspecific ${ }^{15}$.

Increased plasma homocysteine concentration (can be inherited or acquired) are associated with increased risk of myocardial infarction, stroke and venous thrombosis ${ }^{16}$. Our patient had normal plasma homocysteine level.

Deficiency of protein $\mathrm{C}$ and protein S and antithrombin III are some of primary (inherited) thrombophilic conditions and they are sometimes considered in patients who have recurrent $\mathrm{VTE}^{17}$. These tests were within normal range in our patient.

On the CT scan of abdomen our patient presented with DVT of bilateral iliac veins extending to lower end of IVC. These findings fall under the category of ilio-femoral DVT which is defined as thrombosis involving the iliac and/or common femoral veins with or without extension to $\mathrm{IVC}^{18}$. Interestingly our patient did not show any sign/ symptoms of DVT on his right lower extremity.

CT angiogram of chest showed thrombus in right and left pulmonary arteries and large left pleural effusion. Subsequent aspiration of pleural fluid was found to be grossly hemorrhagic. A wedge shaped density at left lower chest on initial X-ray (a second similar density was noted on right chest $\mathrm{X}$ ray later) could indicate presence of pulmonary infarction. It may be responsible for hemorrhagic pleural effusion. But low molecular weight heparin could have a role to play in formation of hemorrhagic pleural effusion. It should be noted dual pulmonary circulation arises from both pulmonary and bronchial arteries. That is why, pulmonary infarction as result of PE is not common. Pleural effusion from $\mathrm{PE}$ are almost always exudative with increase in red cells and leukocytes ${ }^{19}$. Grossly hemorrhagic pleural fluid as found in our case is not common.

Our patient traveled by air from Bahrain to Bangladesh, a distance of $4000 \mathrm{~km}$ with a period of forced immobilization for about nine hours (including transit) and he became symptomatic of PE. Literatures suggest that there is a significant correlation between long distance air travel and occurrence of PE. According to one study risk of PE significantly increased when travel distance exceeded 5000 k.m. resulting in 1.5 cases per million passengers $(\mathrm{p}<0.001)^{20}$. The total incidences of PE reached 4.77 cases per million passengers for distances greater than $10000 \mathrm{~km}$ traveled according to this study.

In conclusion our case illustrates a diagnostic challenge for $\mathrm{PE}$ with its protracted course of illness. There are several 
noticeable risk factors which include strong risk factor like recent amputation of lower extremity, moderate risk factor like suspected thombophilia suggested by recurrent DVT and recent arterial thombo embolism of lower extremity and finally weak risk factors like recent prolonged bed rest after surgery in the hospital followed by long distance air travel. Last but not the least, prevention as well as prompt identification of risk factors will definitely play a great role in minimizing incidence of PE.

\section{References :}

1. Tapson VF, Acute Pulmonary Embolism, NEJM March 6, 2008; $358: 1037-52$

2. Owusu I. A Case Report Of Bilateral Pulmonary Embolism Presenting To Komfo Anokye Teaching Hospital, Kumasi. The Internet Journal of Health. 11(1)5028.

3. Sandler DA, Martin JF. Autopsy proven pulmonary embolism in hospital patients: are we detecting enough deep vein thrombosis? J R Soc Med. 1989 Apr; 82(4):203-5.

4. Lavorini F, Di Bello V, De Rimini ML et al. Diagnosis and treatment of pulmonary embolism: a multidisciplinary approach. Multidisciplinary Respiratory Medicine 2013, 8:75.

5. Virchow R. Phlogose und Thromboseim Gefäbsytem : Gesammelte Abhandlangenzur Wissenschaftlichen medizin. Frankfurt, Germany: Staatsdruckerei. 1856

6. Anderson FAJr, Wheeler HB Physician practices in the management of venous thromboembolism: a community-wide survey. J Vasc Surg. 1992; 16: 707-714.

7. Anderson FAJr, Spencer FA. Risk Factors for Venous Thromboembolism Circulation.2003; 107: I-9-I-16.

8. Ortel TI. Thrombosis and anti phospholipid syndrome. Hematology 2005, January 1, 2005 vol. 2005 no. 1: 462-468.

9. Asherson RA and Harris EN. Anticardiolipin antibodies-clinical associations, Postgraduate Medical Journal 1986; 62:1081-1087.

10. Righini M, Anjesky D, Roy PM, et al, Clinical usefulness of D-dimer depending on clinical probability and cutoff value in outpatients with suspected pulmonary embolism. Arch Intern Med. 2004; 164: 2483-7.
11. Stein PD, Hull RD, Patel KC, et al. D-dimer for the exclusion of acute venous thrombosis and pulmonary embolism: a systematic review. Ann Intern Med.2004 ;140 (8):589-602.http://www.nejm.org/toc/nejm/345/11/

12. Wells PS, Anderson DR, Rodger $M$ et al. Excluding pulmonary embolism at the bedside without diagnostic imaging: Management of patients with suspected pulmonary embolism presenting to the emergency department by using a simple clinical model and D-dimer. Annals Intern Med 2001; 135:98-107.

13. Le- Gal G, Righini M, Roy PM, et al, Prediction of Pulmonary Embolism in the Emergency Department: The Revised Geneva Score. Ann Intern Med. 2006;144:165-71.

14. Jaff MR, McMurtry MS, Archer SL, et al. American Heart Association Council on Cardiopulmonary, Critical Care, Perioperative and Resuscitation; American Heart Association Council on Peripheral Vascular Disease, and American Heart association Council on Arteriosclerosis, Thrombosis and Vascular Biology, Management of Massive and Submassive Pulmonary Embolism, Iliofemoral Deep Vein Thrombosis, and Chronic Thromboembolic Pulmonary Hypertension: A Scientific Statement From the American Heart Association. Circulation. 2011; 123: 1788-1830.

15. Stein PD, Terrain ML, Hales CA, et al. Clinical, laboratory, roentgeno-graphic, and electrocardiographic findings in patients with acute pulmonary embolism and no pre-existing cardiac or pulmonary disease. Chest. 1991; 100(3):598-603.

16. Hortin GL. Homocysteine: Clinical significance and laboratory measurement. Lab. Med.2006 37(9), 551-553

17. Merriman L, Greaves M. Testing for thrombophilia: an evidence-based approach, Postgrad Med J. 2006 Nov; 82 (973):699-704.

18. Liu D, Peterson E, Dooner J et al. Diagnosis and management of iliofemoral deep vein thrombosis: clinical practice guideline. CMAJ. 2015;187(17):1288-96.

19. Agarwal R, Singh N, Gupta D. Pleural Effusions Associated with Pulmonary Thromboembolism: A Systematic Review. Indian J Chest Dis Allied Sci. 2009;51(3):159-64.

20. Lapostolle F, Surget V, Borron SW, et al. Severe Pulmonary Embolism Associated with Air Travel .N Engl J Med 2001; 345:779-783. 